because they were dependent on a place becoming available elsewhere. This was unfortunate as the wait was very frustrating for residents and staff, and the clinical state of some residents seems to regress a little as a consequence. Since their discharge these people have remained dependent on help and support from staff to varying degrees. At first this was provided by staff of the hostel ward but when the unit closed a support team was established in order to continue with this work (Colgan \& Bridges, 1990).

\section{Concluding comment}

Although High Elms was not an ideal building as a hostel ward, the majority of patients admitted were able to benefit from the form of rehabilitation provided by its staff. Those who moved to the more homely purpose-built Anson Road Project have made further progress. From our experience the hostel ward continues to have an important place within the spectrum of services needed for people with chronic psychiatric disorders. However, it has its limitation, particularly with regards to the management of some behaviourally disturbed people. It has also proved to be an inappropriate form of care for those who are against being there. Unfortunately patients returning to hospital on a permanent basis had to join other long-stay patients on acute admission wards because of the lack of more appropriate hospital-based facilities for them (Wing, 1990).

\section{References}

Abrams, M. (1988) Psychiatric morbidity of long stay hospital population (letter) British Medical Journal, 297, 1334.

Colgan, S. \& Bridges, K. (1990) The development of a community support service for the chronically mentally ill in an inner city health district. Psychiatric Bulletin, 14, 710-711.

GareTY, P. A., Afele, H. K. \& IsaAcs, A. D. (1988) A hostel ward for new long-stay psychiatric patients: the careers of the first 10 years' residents. Bulletin of the Royal College of Psychiatrists, 12, 183-186.

Hyde, C., Bridges, K., Goldberg, D., Lowson, K., STERLING, C. \& FARAGHER, B. (1987) The evaluation of a hostel ward. A controlled study using modified costbenefit analysis. British Journal of Psychiatry, 151, 805-812.

Wing, K. J. (1990) The functions of asylums. British Journal of Psychiatry, 157, 822-827.

\title{
Are your casenotes perfect?
}

\author{
Sara Cunningham, Registrar in Psychogeriatrics, Mossley Hill Hospital, \\ Park Avenue, Liverpool L18 8BU
}

Working for Patients (1989) demonstrates the Government's intention to "encourage all Royal Colleges to make participation in medical audit a condition of a hospital unit being allowed to train doctors", suggesting this may also become a requirement for psychiatric trainees. The Psychiatry Department at Fazakerley Hospital moved into the rather uncharted territory of clinical audit with monthly unit audit meetings in 1989. At each meeting randomly selected cases are reviewed and data presented which are relevant to clinical practice on the unit. The majority of topic audits have been presented by trainees with support from consultants. Most Colleges agree that casenotes should be available for scrutiny (Warden, 1988). Consequently I undertook a casenote audit.

The "perfect casenote" should serve four main functions: informative - containing patient data and chronological details of events; legal - being the only written document recording the doctor's management of an individual case; communicative enabling transfer of information between and/or within specialties, professionals and other hospitals; and storage-storage of information for future reference.

\section{The study}

The audit was carried out in the psychiatric in-patient unit of a district general hospital with 125 acute beds (approximately 15 beds per consultant). Disciplines incorporated were general adult psychiatry and functional psychogeriatrics, reflecting the practice of $\mathbf{2 0}$ doctors from consultant to SHO.

Four casenotes were selected blind from each ward by nursing staff. Duration of admission ranged from 
two weeks to greater than eight weeks (median four to eight weeks). The auditor's consultant was excluded to prevent bias.

After consideration of essential requirements, the auditor prepared a "perfect casenote" to act as a standard. This was divided into four sections: patient information; details of admission; ward review; and general impression.

Each section was broken into discrete items and a simple 3 point scale measured how each item had been recorded, i.e. (1) clearly present; (2) implied (information present but incomplete); and (3) clearly absent.

Global ratings of readability and legibility were also included. Emphasis was on the initial admission note but review by the clinical team was also assessed.

\section{Findings}

There were eleven female and nine male patients with clinical diagnoses of: schizophrenia (10), depression (6), mania (3) and organic (1). The patient information section which rated basic demographic data was comprehensive, typed, and easily accessible. Almost all the information was present in 19/20 (95\%).

Admission details showed the legal status was clear where the patient was detained under a Section of Mental Health Act 1983 but absent when the admission was informal. Presenting complaint and its history were never absent but often difficult to read or left one unclear of events. Past medical history (PMH) was absent in 4/20 (20\%) and past psychiatric history (PPH) in $1 / 20(5 \%)$. PPH lacked details in $9 / 20(45 \%)$ although in some a later summary clarified the situation. As history-taking progressed information became less frequent and less clear: $17 / 20(85 \%)$ noted drugs on admission, $11 / 20$ (55\%) clearly stated family history, personal history, alcohol and tobacco consumption. Social history was absent in $8 / 20(40 \%)$ and clear in only $5 / 20$ (25\%). Physical system review was absent in all casenotes. Previous case summaries were a useful reference and the handwritten entries were clearer to read with double spacing.

In mental state examinations, appearance and behaviour, mood and speech were well covered. Thought was less well assessed, clear in only 13/20 $(65 \%)$, and some doctors seemed unsure of the terms they were using, rarely making definitive statements. Perception was not recorded in 5/20 (25\%) primarily those with affective disorders. A more worrying finding was that suicide risk had not been recorded and possibly not assessed in 10/20. Cognitive assessment was done in only half the casenotes and comments on insight were often unhelpful, e.g. nil, none, good! One set of casenotes had no entry for mental state, despite ten days having elapsed since the previous admission during which the patient had absconded and been readmitted on Section 3 MHA.

Physical examination was performed in all casenotes. Initial management plans had no comment on nursing observations required for $10 / 20(50 \%)$ of cases and no differential diagnosis in $5 / 20(25 \%)$. Proposed treatment regimes to be instituted after admission were unclear or the dosages of medication not stated.

In the ward review section, description of progress was good but review of diagnosis was absent in $7 / 20$ $(35 \%)$. In $8 / 20(40 \%)$ there was no evidence of informant information. Treatment changes were documented but unclear and management plans present in all but one.

Global rating showed that in $9 / 20(45 \%)$ legibility was poor and only $12 / 20(60 \%)$ were easily readable. Legibility and readability were clearly linked. Most entries were signed but staff posts were not commonly stated. Finally in $15 / 20(75 \%)$ there was no evidence that any information had been given to relatives.

\section{Comment}

The process of audit should be relevant, objective, quantified, repeatable and able to effect appropriate change in organisation of the service and clinical practice (Shaw, 1989). A casenote study by Ellis et al (1987) concluded that for peer review to be effective not only do issues need to be discussed but also reinforced at a later date.

Although we anticipated that our casenotes would not be "perfect", the audit served the purpose of identifying poorly presented areas and this focus of attention is necessary to change practices. It is more useful to show that suicidal ideation, patients' legal status or PPH are not being recorded than to simply say casenotes are incomplete and unsatisfactorily kept.

The meeting concluded with the following recommendations: more attention was required by all medical personnel to their casenote entries; each new intake of trainees should be informed of the audit findings; casenote audit applying the same method should be repeated, most usefully toward the end of trainees' time on the unit, to evaluate positive change.

Senior clinicians were as guilty of poor performance as trainees and repeat audits will remind consultants of their obligations in this area. Casenote audit is clinically relevant and data accessible. It has the potential to improve our practice and we would recommend its routine use.

\section{Acknowledgement}

I would like to thank Dr D. N. Anderson for his helpful advice and support in preparing this article. 


\section{References}

Ellis, P. M., Mellsop, K. A., Peace, K. A. \& Wilson, J. M. (1987) Peer review as an aid to improving the completeness of psychiatric casenotes. Medical Education, 21, 493-497.

Secretaries of State for Health, Wales, Northern IRELAND AND SCOTLAND (1989) Working for Patients. London: HMSO.
Shaw, C. D. \& Costain, D. W. (1989) Guidelines for medical audit: seven principles. British Medical Journal, 299, 498-499.

WARDEN, J. (1988) All for audit now. British Medical Journal, $297,1569$.

Psychiatric Bulletin (1991), 15, 674-675

\section{Suicide}

\section{J. Rossiter, Senior Registrar, Tone Vale Hospital, Taunton, Somerset TA4 1DB}

In an attempt to discover what can be learnt from records kept on suicide victims, all data collected by one health district about suicides occurring in patients in close contact with psychiatric care over four years were examined.

These comprised 12 in-patients, six recently discharged patients, and four patients in close contact with the CMHT. They were treated by eight different consultants and CMHTs. No one team had an excess of deaths.

In this district, as in many others, there is a policy that following a suicide, the care team should meet as soon as possible to review the case. The stated aim of this meeting is to offer staff support and constructive self-criticism. Following this a report is sent to the Mental Health Unit Manager. An examination of the reports of 22 suicides (certified as such by the Coroners' courts) revealed that in all cases the care plan was seen as "appropriate". Only six cases had been recognised as "at risk of suicide", that is in most cases the risk had been considered low and ongoing. Although all patients had had previous contact with the psychiatric services, often over a long period of time, in only three cases was any attempt made to suggest why the suicide had occurred now. In only six cases were recommendations for change made. These all concerned measures to increase the supervision of the patient by the care team.

While it is impossible to say whether or not these written reports are a reasonable reflection of what was said or thought at the time, they are the only documentary evidence of the cases that are kept and can be studied. They were produced following the mental health team reviews and thus must represent some consensus view on how the case was seen. Private thoughts and reflections always remain so. Thus although the individual may have learnt something from the suicide no-one else will have access to that new understanding. Learning from personal experience cannot be the best way to increase knowledge about the risk factors for suicide. Is it reasonable to expect more useful information?

We are members of the 'caring professions'. The unexpected death of a patient leaves all staff to a greater or lesser extent shocked and distressed.

We know from work with bereaved relatives (Murray Parkes, 1985) that grief is complicated when a death is unexpected, inexplicable, and when the relative in some way feels responsible. One way to help staff through their grief is to attribute the suicide to within-patient factors, i.e. his/her illness, and to assure everyone that all possible steps had been taken. While such statements cannot be construed as constructive self-criticism, they may be absolutely essential at the time to enable staff to continue working with an often difficult patient/client group. Placing the problem solely with the patient can reassure staff that their judgement was correct, and their caring skills remain intact. Such statements may also be what many people want to hear. Many professionals are uncertain about their job security as mental hospitals close and units are relocated in the community. Managers are fearful of litiginous relatives and relatives themselves may feel that the professionals' judgement "nothing more could have been done" absolves them from any guilt.

The danger is that nothing new is learnt. Many years of research have produced good epidemiological information about groups of patients at risk, but are of little use in defining individuals at risk (Hawton, 1987). Goh et al (1989) recently called for more research into interpersonal and environmental aspects of suicide. This information can be given only by those people who knew the patient well. It can be collected only if the informants can recall and discuss their memory of their patient and their interactions 